\title{
Elemental Distribution in Individual Rain Droplets Determined by a Combination of the Replication Method and the Synchrotoron Radiation X-ray Fluorescence Microprobe Technique
}

\author{
Chang-Jin Ma, ${ }^{* 1 \dagger}$ Susumu Tohno, ${ }^{* 2}$ Mikio Kasahara, ${ }^{* 3}$ and Shinjiro HayaKawa*4 \\ *1 Department of Environmental Science, Fukuoka Women's University, Higashi-ku, Fukuoka 813-8529, Japan \\ *2 Graduate School of Energy Science, Kyoto University, Uji, Kyoto 611-0011, Japan \\ *3 Institute of Science and Technology Research, Chubu University, Kasugai, Aichi 487-8501, Japan \\ *4 Graduate School of Engineering, Hiroshima University, Higashi-Hiroshima, Hiroshima 739-8527, Japan
}

\begin{abstract}
The main objective of the present study is to specify the chemical properties of individual rain droplets. For this purpose, we have combined the collodion replication method and the SR-XRF microprobe technique. The dry residual materials retained in a single rain droplet, which correspond to the former cloud condensation nuclei and the scavenged particles during droplet falling, were successively reconstructed by the multiple elemental maps using SR-XRF microprobe analytical system. Also the SR-XRF microprobe system allows us to quantify the masses of ultra trace elements in residues of individual rain droplet with fg level. The proposed combination method in the present study is found to be helpful to understand the physicochemical properties of individual rain droplets.
\end{abstract}

(Received August 22, 2005; Accecpted December 21, 2005)

\section{Introduction}

The chemical processes in the atmosphere as the washout of particles and gases are not sufficiently described by the usual determination of elemental concentration in rain water. The chemical content of raindrops is variable according to many mechanisms such as condensation nuclei inside clouds, pollutant scavenging, collision, coalescence and break-up of falling raindrops, and evaporation. ${ }^{1,2}$ The analysis of rain as bulk phase will lead to a loss of detailed information, because the chemical content of raindrops depends on the drop size. ${ }^{1,3}$ But in general, the analysis of rain sample was nearly always carried out on the assumption that individual raindrops forming the bulk phase have the same concentrations of trace compounds as the bulk rainwater. This assumption may be justified considering average effects of rainwater on the biosphere, soil, etc. The effects of raindrops which may have concentrations of trace elements, which are much higher than the bulk rainwater, are completely neglected by this assumption. Therefore, to get the detailed information on the chemical content of raindrops and the scavenging processes of atmospheric pollutants by rain events, one must perform the sampling and analysis of individual raindrops.

The lower falling velocities of rain droplets make them remain in the atmosphere for a longer time than raindrops. This longer lifetime also causes severe evaporation during their fall. Consequently, the evaporated rain droplets with highly concentrated chemical components exert a harmful influence upon our ecosystem.

The chemical properties of individual rain droplets depend on

$\dagger$ To whom correspondence should be addressed.

E-mail: ma@fwu.ac.jp the various interactions of droplets, gases and solid particles. However, the increasing of elemental concentration has its limits because a single rain droplet has a tiny volume. To determine the chemical properties of individual droplets, we need a fully quantitative analytical technique that has exceedingly high sensitivity.

In the present study, through the combination with the collodion replication method for the collection of individual rain droplets and the synchrotoron radiation X-ray fluorescence (SRXRF) microprobe analytical technique, the specific chemical investigation for individual rain droplets could be realized.

\section{Experimental}

Replication of individual rain droplets

Unlike solid specimens, droplets are unstable. Any contact with a solid surface or another droplet makes the droplet disappear. Also it is difficult to collect droplets individually because a single droplet has a tiny volume. For these reasons, $\mathrm{Ma}^{3}$ introduced the collodion replication technique for the collection of individual droplets. The key feature of this technique is indirect droplet sizing from the diameter of droplet replication. The other key feature of the proposed method is convenience without power supply. Additionally, subsequent chemical analysis is possible without any sample pretreatment. In the present study, this collodion film method was applied to isolation of individual rain droplets and their subsequent chemical analysis. However, unfortunately, this collodion film method did not allow to sampling of raindrops larger than 2.1 $\mathrm{mm}$ diameter because of breakup of large raindrops when they collide with a collodion film surface. ${ }^{4}$ Therfore, in the present study, the collodion film method was applied to the sampling of 


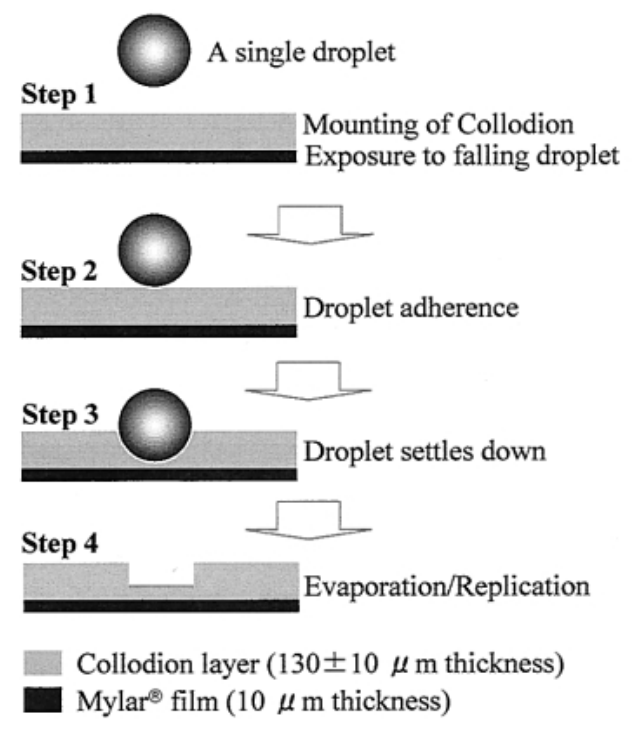

Fig. 1 Replica-forming processes of individual rain droplets on the thin collodion layer mounted onto Mylar ${ }^{\circledR}$ film.

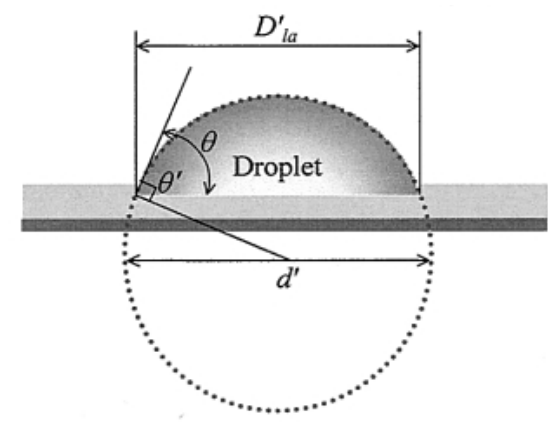

Collodion layer $(130 \pm 10 \mu \mathrm{m}$ thickness $)$

Mylar ${ }^{\circledast}$ (10 $\mu$ m thickness)

Fig. 2 Calculation of the real diameter of flattened droplets on the collodion layer surface.

fine droplets falling a drizzle.

Since the droplet replication process was already described in former studies, ${ }^{4,5}$ it is noted here only briefly. Figure 1 illustrates the replica forming processes of individual rain droplets on the thin collodion layer mounted onto Mylar ${ }^{\circledR}$ (polyethylenetelephtalate) which is an extraordinarily strong polyester film. The thickness of Mylar ${ }^{\circledR}$ used as the backing material of the thin collodion layer is $10 \mu \mathrm{m}$. A viscous collodion solution was mounted onto the Mylar ${ }^{\circledR}$ just before exposure to the falling droplets (Step 1 in Fig. 1). Rain droplets adhere onto the thin layer of collodion film (Step 2 in Fig. 1), and then they gently settled without bounce-off (Step 3 in Fig. 1). After evaporation of alcohol and ether from collodion, a thin film containing the replications of individual rain droplets was left (Step 4 in Fig. 1). By using this replication technique, which is one of passive sampling methods without any pumps, a field sampling was conducted at a height of $20 \mathrm{~m}$ above ground level of the Kyoto University building located in Uji, Japan during the time period between 13:30 - 14:10 on 29 Nov. 2001. It drizzled from afternoon till night. About 50 separated individual rain droplets were collected on 10 sheets of collodion film. Before the field measurement, the experimental

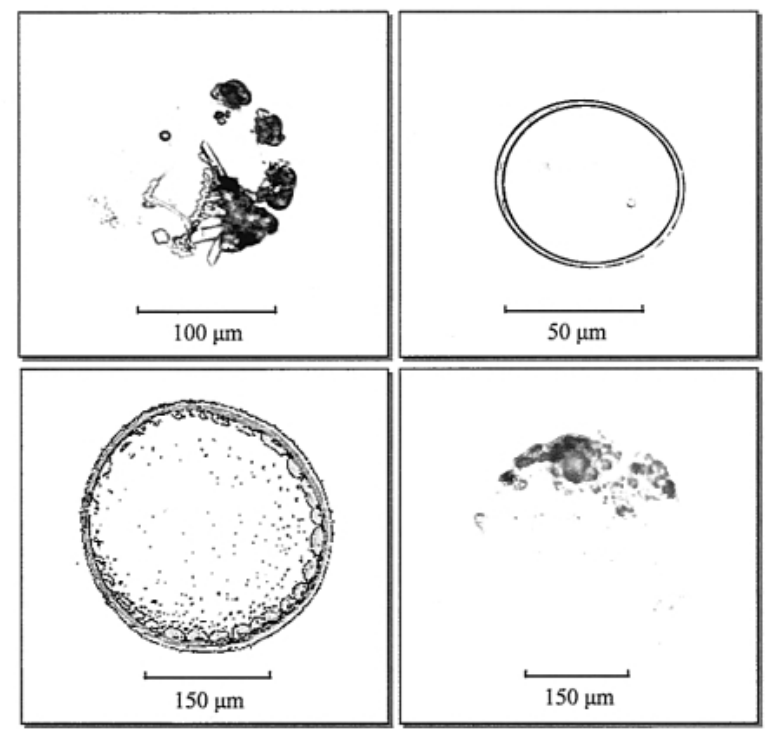

Photo 1 Images of residues retained in individual rain drops captured on the collodion film.

apparatuses such as petridishes for loading Mylar $^{\circledR}$ and collodion solution were left outside and pre-conditioned to the ambient outdoor temperature.

Chemical specification of droplets by X-ray fluorescence (XRF)

In the present study, the XRF microprobe system equipped at Super Photon ring-8 GeV (SPring-8), BL-37XU, was applied to the quantification analysis of ultra trace elements in residues of individual rain droplets. This method can be successfully used to carry out the reconstruction of elemental map and the quantification analysis for multiple elements with fg level sensitivity. ${ }^{6}$ BL-37XU is a hard X-ray undulator beamline that is mainly used for studies of X-ray micro-spectrochemical analysis such as XRF imaging, XAFS, TXRF and XRF holography. To make the narrow X-ray beam with micro scale, a pair of elliptical mirrors (KB mirror) was installed in the optical path. The beam size obtained with this experiment was $4 \mu \mathrm{m}$.

After allocating the target portion by an optical microscope, a sample was placed on the XY stage in a vacuum chamber of the XRF analyzer installed in hutch 1, BL-37XU. A $10 \mathrm{keV}$ X-ray microbeam was employed for the measurement of individual rain droplets. By scanning the microbeam on the surface of droplet replica, it is possible to obtain spatial distribution of trace elements in scanning area $200 \times 200 \mu \mathrm{m}^{2}$. The intensity of the incident $\mathrm{X}$-rays was monitored by an ionization chamber. The fluorescence X-rays were recorded with a $\mathrm{Si}(\mathrm{Li})$ detector placed in the electron orbit plane of the storage ring and mounted at $90^{\circ}$ to the incident X-rays to minimize background caused by the scattering. To realize elemental quantification, we measured XRF spectra of the selected portions and the blank of backing material (Mylar ${ }^{\circledR}$ film) with the data acquisition time of $200 \mathrm{~s}$.

\section{Results and Discussion}

Replicated rain droplets

Photo 1 illustrates the images of residues retained in four types of individual rain drops captured on collodion film. According to the visual property, four kinds of droplet replicas 
Table 1 Calculated ratio $R\left(R=D_{\mathrm{la}} / D_{\text {la }}^{\prime}\right)$ with each contact angle, $\theta$ (in Fig. 2)

\begin{tabular}{cccc}
\hline Contact angle $(\theta)$ & Ratio $(R)$ & Contact angle $(\theta)$ & Ratio $(R)$ \\
\hline 5 & 0.254 & 50 & 0.572 \\
10 & 0.320 & 55 & 0.597 \\
15 & 0.368 & 60 & 0.622 \\
20 & 0.406 & 65 & 0.647 \\
25 & 0.439 & 70 & 0.673 \\
30 & 0.469 & 75 & 0.701 \\
35 & 0.496 & 80 & 0.730 \\
40 & 0.522 & 85 & 0.760 \\
45 & 0.548 & 90 & 0.794 \\
\hline
\end{tabular}

were differentiated among 50 droplet replicas. The residual materials retained in four types of rain drops displayed in Photo 1 as the examples were irradiated by XRF microbeam.

Rain droplet, which can be defined as the fine raindrop, are usually about one-fifth of a millimeter across; fully fledged raindrops measure a couple of millimeters or so. A previous quantitative theoretical and experimental investigation ${ }^{7}$ showed that at $1000 \mathrm{hPa}$ and $20^{\circ} \mathrm{C}$ drops of equivalent radius 100,200 , $300,400,500,600,700,800,900,1000$ and $2000 \mu \mathrm{m}$ require $0.15,0.71,1.6,2.8,3.9,5.2,6.3,7.3,8.4,9.5$ and $14.0 \mathrm{~m}$ for accelerating to $99 \%$ of their terminal velocity, respectively. According to this result, smaller raindrops show lower terminal velocities. Smaller raindrop (drizzle) falls to the ground at a leisurely speed of half a meter or so per second. Because of air currents, drizzle droplets seem almost to hover in the air. This makes drizzle droplets remain for a longer time in the atmosphere and also this longer lifetime causes severe evaporation during their fall. If evaporation occurs, the concentration in drizzle droplets increases more than the concentration in raindrops. Consequently, although drizzle is a less frequent than rain, the concentration of chemical compositions in drizzle droplets will be higher than that of rain. Finally owing to their high chemical content, drizzle droplets play an important role in many environmental processes.

As shown in Photo 1, the size range of droplet replicas is up to around $300 \mu \mathrm{m}$. Also, some fine rain droplets with $30-70 \mu \mathrm{m}$ diameters like right the upper one in Photo 1 were replicated. These small droplets are so small that they are carried by the wind and they do not really strike the ground by free falling. In the droplet replicas formed on collodion film, the residual materials which are the cluster of insoluble/involatile materials retained in droplets were to be the target of chemical analysis.

\section{Diameter calculation for spherical droplet}

As mentioned above, since the chemical content of droplets depends on the droplet size, an accurate droplet sizing is critical for the estimation of droplet size-dependence of elemental concentration. Liquid droplets collected onto collodion film are flattened and increased in size in the field of microscope. Hence, it is necessary to calculate the initial droplet diameter. Figure 2 presents the calculation method of the real diameter of flattened droplets on collodion layer surface. Because the liquid droplets adhered to the surface of collodion film are not spherical in shape, their apparent diameter, $D_{\text {la }}^{\prime}$, measured by digital microscope (KEYENCE, VH-7000) is larger than their real diameter $D_{\text {la }}$ in the airborne stage. In order to use the microscopic technique for sizing liquid droplets, the ratio

$$
R=\frac{D_{\mathrm{la}}}{D_{\mathrm{la}}^{\prime}}
$$

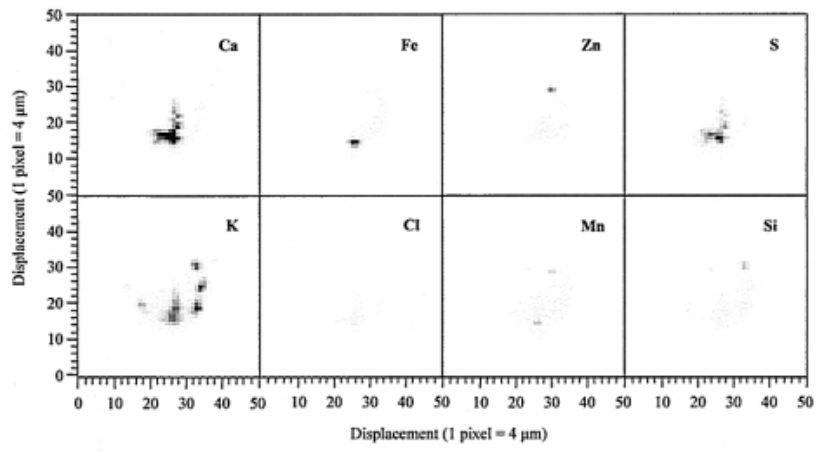

Fig. 3 XRF elemental maps of a rain residue imaged in Photo 1 (upper left). $10 \mathrm{keV} \mathrm{X-ray} \mathrm{microbeam} \mathrm{with} 4 \mu \mathrm{m}$ beam size was employed. Scanning area is $200 \times 200 \mu \mathrm{m}^{2}$.

must be known. The ratio $R$ is seen to be a function of the contact angle $\theta$ between the liquid droplet and the collodion film surface (in Fig. 2). For small droplet diameter, gravitational effects are negligible. The collected liquid droplet forms a spherical section with the volume $V$,

$$
V=\pi\left[\frac{D_{\mathrm{la}}^{\prime}}{2}\right]^{3}\left[\frac{\frac{2}{3}-\cos \theta+\frac{1}{3}-\cos \theta^{3}}{\sin \theta^{3}}\right]
$$

Liu et al. ${ }^{8}$ measured the volume of a droplet forming a spherical section using Eq. (2). Their experimental values of $R$ (Eq. (1)) compared with the theoretical values based on contact angle $(\theta)$ measurements showed good agreement.

Since the volume of spherical liquid aerosol with diameter $D_{\text {la }}$ is

$$
V=\frac{4}{3} \pi\left[\frac{D_{\mathrm{la}}}{2}\right] \text {. }
$$

The ratio $R$ can be rearranged from Eqs. (2) and (3)

$$
R=\frac{D_{\mathrm{la}}}{D_{\mathrm{la}}^{\prime}}=\frac{\left[\frac{3}{4}\left(\frac{2}{3}-\cos \theta+\frac{1}{3}-\cos \theta^{3}\right)\right]^{1 / 3}}{\sin \theta} .
$$

Table 1 lists the values of $R$ calculated from Eq. (4). The contact angles $(\theta)$ and the sizes of $D_{\text {la }}^{\prime}$ were measured with a digital microscope which has multiple functions. The contact angles $(\theta)$ for nearly all of rain droplets collected in the present study were around $90^{\circ}$, consequently the original diameter of liquid rain on collodion film, $D_{\text {la }}$, was calculated by $0.794 \times D_{\text {la }}^{\prime}$ (in Fig. 2). The number of 0.794 is the ratio $(R)$ when the contact angles $(\theta)$ is $90^{\circ}$.

\section{Reconstruction of elemental maps}

By natural evaporation of the liquid water and the volatile substances at room temperature, finally the non-volatile dry residues are released. These residual materials were to be the target of chemical analysis using the XRF microprobe installed at SPring-8. Figure 3 shows the XRF elemental maps of a rain residue imaged at upper left in Photo 1 . The replica diameter of this rain and the calculated real diameter as a spherical droplet from Eq. (4) are $140 \mu \mathrm{m}$ and $111 \mu \mathrm{m}$, respectively. The $10 \mathrm{keV}$ $\mathrm{X}$-ray microbeam with $4 \mu \mathrm{m}$ beam size was employed over a scan area of $200 \times 200 \mu \mathrm{m}^{2}$. Soil derived components such as 


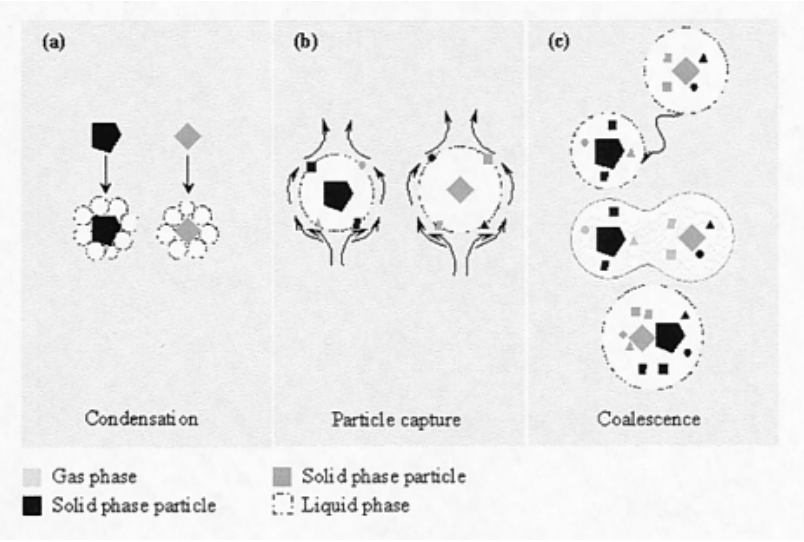

Fig. 4 Schematic explanation of elemental inhomogeneous in a rain droplet.

$\mathrm{Ca}, \mathrm{Fe}, \mathrm{K}$, and $\mathrm{Si}$ are clearly associated and the artificial trace element like $\mathrm{Zn}$ and $\mathrm{Mn}$ as well as $\mathrm{S}$ appear to be retained in a single rain droplet. These multiple XRF elemental maps show that the composition of retained particle cluster in a single droplet replica is not spatially homogeneous. The inhomogeneous chemical distribution of local structures around droplet replica was also found in three other droplet replicas. Due to a limited resolution ( $4 \mu \mathrm{m}$ beam size) of the XRF focusing system, detailed structural information such as the resolution of elemental maps for separate individual particles captured in a rain droplet was not obtained. However the XRF elemental maps for multiple elements indicate that numerous particles containing different chemical composition are retained in a single rain droplet.

Figure 4 schematically explains the expected mechanisms for the elemental inhomogeneous in a rain droplet. When water vapors condense on a particle (condensation nuclei), the particle diameter and shape are changed ((a) in Fig. 4). The ability of a given particle to serve as a nucleus for water droplet formation will depend on its size, chemical composition, and the local supersaturation. ${ }^{9}$ The differences of elemental distribution in rain droplets arise from differences in the composition of the condensation nuclei. The soluble gases and various particles with different chemical components are transferred into falling droplets below a cloud ((b) in Fig. 4). Particles can be scavenged by droplets in the atmosphere through the following processes; Brownian diffusion through coagulation of the particles with radius less than $0.1 \mu \mathrm{m}$ with wet droplets, collision of particles with wet droplets by phoretic forces such as thermophoresis and diffusiophoresis, inertial impaction of particles on droplets, and electrical forces which can promote precipitation. ${ }^{10}$ Larger droplets fall faster than smaller ones, resulting in the larger droplets overtaking the smaller ones, colliding and coalescing with them ((c) in Fig. 4). This process is growth by accretion, sometimes also called gravitational coagulation, coalescence, or collisional growth. ${ }^{9}$ Through this coalescence of droplets which are containing different particles, the elemental inhomogenity of droplets collected on the ground surface might be caused.

\section{XRF elemental spectra and masses}

Figure 5 displays the XRF spectra for three points marked at a replica image. Two different positions on a droplet replica and one position on blank collodion film were the subjected to individual spectrum measurements for XRF analysis. As suggested by XRF elemental maps of a whole rain droplet, soil

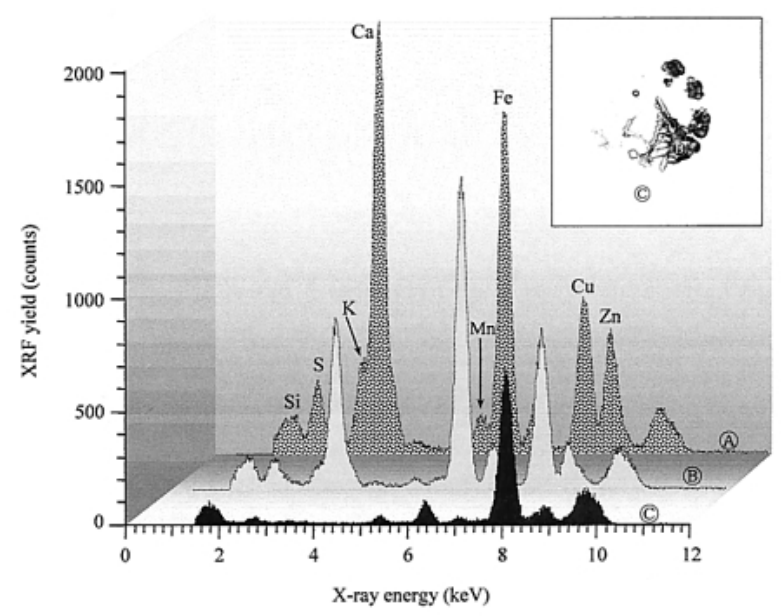

Fig. 5 XRF spectra for three positions described at a replica image. XRF counts were counted for $100 \mathrm{~s}$. Position (C) means blank of collodion film.

originated components show relatively distinct peaks. From these XRF spectra for different positions in a single droplet replica, it can be said that many elemental components coexist with several soil and anthropogenic originated components. Also XRF spectra for two portions (A) and (B) in Fig. 5) show the position-to-position component variation in a single droplet. Unfortunately, the XRF spectrum for $\mathrm{Cu}$ was overestimated because the sample holder of XRF microprobe analytical system contains $\mathrm{Cu}$. Cu peaks of positions (A) and (B) in Fig. 5 resolved with equal height to collodion film blank's one indicate that $\mathrm{Cu}$ at positions (A) and (B) was derived from collodion film.

The elemental mass thicknesses and masses calculated by XRF analysis for the three positions in a single rain droplet replica imaged in Fig. 5 are summarized in Table 2. The areal density of multiple elements $\left(\mu \mathrm{g} \mathrm{cm}^{-2}\right)$ was calculated from the comparisons of the gross signals between the sample and the sensitivity curve $\left(y=2.039 e^{0.3225 x}, R^{2}=0.9799\right)$ drawn from $\mathrm{Si}$ to $\mathrm{Zn}$ with the fivefold reference $(\mathrm{Si}, \mathrm{Ti}, \mathrm{Cr}, \mathrm{Fe}, \mathrm{Ni}$ ) thin film. These five representative materials were evaporated on Kapton foil of $10 \mu \mathrm{m}$ in thickness. The thicknesses of $\mathrm{Si}, \mathrm{Ti}, \mathrm{Cr}, \mathrm{Fe}$, and $\mathrm{Ni}$ coated on Kapton foil are 18, 6, 6.9, 7.0, and $5.3 \mathrm{~nm}$, respectively. Elemental masses (pg) for different positions in a rain replica were calculated from the beam size and areal density of elements $\left(\mu \mathrm{g} \mathrm{cm}^{-2}\right)$. Details of the calibration of the $\mathrm{X}$-ray microprobe system to yield quantitative measures of elemental mass in individual particles including the effects of the self-absorption were described elsewhere. ${ }^{6}$

As the major crustal originated components, the mass of $\mathrm{Ca}$ and $\mathrm{Fe}$ show higher mass levels than those of other elements at portions (A) and (B) (in Fig. 5). They are in the range of $0.39 \mathrm{pg}$ to $1.06 \mathrm{pg}$ (average $0.73 \mathrm{pg}$ ) and $0.24 \mathrm{pg}$ to $0.54 \mathrm{pg}$ (average $0.39 \mathrm{pg}$ ), respectively. The man-made components $\mathrm{Mn}$ and $\mathrm{Zn}$ have poor mass. The significant mass levels for crustal components indicate that a large number of crustal particles were incorporated into rain droplets collected during our sampling campaign.

\section{Conclusions}

To obtain the morphological information on individual rain droplets and their chemical properties, we proposed a reliable technology in the present study. It should be concluded, from 
Table 2 Quantitative elemental masses calculated by XRF analysis for three positions in a rain replica displayed in Fig. 5

\begin{tabular}{|c|c|c|c|c|c|c|}
\hline \multirow{2}{*}{ Element } & \multicolumn{2}{|l|}{ Position (A) } & \multicolumn{2}{|l|}{ Position (B) } & \multicolumn{2}{|l|}{ Position (C) ${ }^{a}$} \\
\hline & Mass thickness $/ \mu \mathrm{g} \mathrm{cm}^{-2}$ & Mass/pg & Mass thickness $/ \mu \mathrm{g} \mathrm{cm}^{-2}$ & Mass/pg & Mass thickness $/ \mu \mathrm{g} \mathrm{cm}^{-2}$ & Mass/fg \\
\hline $\mathrm{S}$ & 0.70 & 0.14 & 0.84 & 0.16 & 0.28 & 55.30 \\
\hline $\mathrm{Ca}$ & 2.00 & 0.39 & 5.41 & 1.06 & 0.33 & 64.14 \\
\hline $\mathrm{Mn}$ & 0.03 & 0.01 & 0.09 & 0.02 & 0.01 & 1.13 \\
\hline $\mathrm{Fe}$ & 1.24 & 0.24 & 2.74 & 0.54 & 0.06 & 11.60 \\
\hline $\mathrm{Zn}$ & 0.03 & 0.01 & 0.11 & 0.02 & 0.01 & 1.46 \\
\hline
\end{tabular}

a. Collodion film blank.

what has been said above, that the combination of the replication method and SR-XRF microprobe technique is undoubtedly a profitable method to study natural removal processes of atmospheric pollutants by droplets. Especially the results obtained through the analysis of individual droplets by SR-XRF microbeam method, such as chemical distribution and mixed elemental state in individual rain droplets, should be helpful to better understand the cloud-nucleation and scavenging processes of pollutants by droplet. Even though the various elemental masses in the residues of rain droplet were determined through the SR-XRF analysis in the present study, further attempts at obtaining absolute element concentration $(\mathrm{w} / \mathrm{v})$ of a whole rain droplet are in progress. Moreover, in order to get a full understanding of droplet formation and its multiple scavenging processes, further field studies considering the chemical determination for a large amount of droplet samples in various rainfall events are being planned.

\section{Acknowledgements}

This study was supported in part by funds from the Grant-inAid for Scientific Research on Priority Areas under Grant No. 14048212 and 14048213 from the Ministry of Education, Culture, Sports, Science and Technology (MEXT), Japan. Also this study was partly carried out under the aid of the program "Establishment of COE on Sustainable-Energy System". The synchrotron radiation experiments were performed at the
SPring-8 with approval of the Japan Synchrotron Radiation Research Institute (JASRI) (Proposal No. 2002B0395-NOS-np, 2002A4029-LM-np). The authors wish to express thanks to all the members of SPring-8, BL-37XU. Finally, the authors also express sincere thanks to Y. Inoguchi for her sampling support.

\section{References}

1. B. Tenberken and K. Bächmann, J. Chromatogr., A, 1996, 755, 121.

2. C.-J. Ma, M. Kasahara, S. Tohno, and T. Kamiya, Water, Air Soil Pollut., 2001, 130, 1601.

3. C.-J. Ma, Ph. D. Thesis, Kyoto University, 2001.

4. C.-J. Ma, M. Kasahara, S. Tohno, and T. Sakai, Atmos. Environ., 2003, 37, 4679.

5. C.-J. Ma, M. Kasahara, S. Tohno, and T. Sakai, J. Jpn. Soc. Atmos. Environ., 2003, 38, 89.

6. S. Hayakawa, N. Ikuta, M. Suzuki, M. Wakatsuki, and T. Hirokawa, J. Syncrotoron Radiation, 2001, 8, 328.

7. P. K. Wang and H. R. Pruppacher, J. Atmos. Sci., 1977, 34, 1664.

8. B. Y. H. Liu, D. Y. H. Pui, and X. Q. Wang, Atmos. Environ., 1982, 16, 563

9. J. H. Seinfeld and S. N. Pandis, "Atmospheric Chemistry and Physics", 1998, John Wiley \& Sons, Inc., 1003.

10. M. J. Pilat and A. Prem, Atmos. Environ., 1976, 10, 13. 